\title{
IDENTIFICAÇ̃̃O DAS REGIÕES PLUVIOMÉTRICAS HOMOGÊNEAS E INÍCIO E FIM DA ESTAÇÃO CHUVOSA NA BACIA DO MÉDIO SÃO FRANCISCO (BRASIL)
}

\author{
Laurizio Emanuel Ribeiro Alves \\ Instituto Nacional de Pesquisas Espaciais \\ laurizio.r@gmail.com \\ Heliofábio Barros Gomes \\ Universidade Federal de Alagoas \\ heliofab@gmail.com \\ Washington Luiz Félix Correia Filho \\ Universidade Federal de Alagoas \\ wlfcfm@gmail.com \\ José Francisco de Oliveira Júnior \\ Universidade Federal de Alagoas \\ jose.junior@icat.ufal.br \\ Luis Gustavo Gonçalves de Gonçalves \\ Instituto Nacional de Pesquisas Espaciais \\ luis.goncalves@inpe.br \\ Dirceu Luis Herdies \\ Instituto Nacional de Pesquisas Espaciais \\ dherdies@gmail.com \\ Fabrício Daniel dos Santos Silva \\ Universidade Federal de Alagoas \\ fabricio.santos@icat.ufal.br
}

\section{RESUMO}

A escassez hídrica resultante da má distribuição espaço-temporal das chuvas gera grandes dificuldades no abastecimento de água para a população da maior parte do semiárido nordestino e regiões adjacentes. Desta maneira, torna-se imprescindível o conhecimento sobre o regime pluviométrico de uma Bacia Hidrográfica como maneira de monitorar ocorrências de seca, neste caso, da Bacia do Médio São Francisco (BMSF), Brasil. Neste trabalho utilizaram-se dados diários de chuvas de 22 estações meteorológicas convencionais do Instituto Nacional de Meteorologia (INMET), localizadas na BMSF e áreas adjacentes, do período de 2001 a 2017. Para a identificação dos padrões de chuva, utilizou-se a análise de Cluster via método de Ward para a obtenção de grupos homogêneos. Em seguida, foi estudada a sazonalidade da bacia, bem como o início (IEC) e fim (FEC) da estação chuvosa via método de Liebmann. O regime de chuvas ao longo da BMSF foi subdividido em três grupos homogêneos (G1, G2 e G3). Além disso, esses grupos apresentaram padrão sazonal de chuva similar, um seco entre os meses de maio e setembro e outro chuvoso entre os meses de outubro a abril. O IEC e FEC se iniciam em meados de outubro e em março, respectivamente.

Palavras-chave: Bacia Hidrográfica. Chuva. Análise de Cluster.

\section{IDENTIFICATION OF HOMOGENEOUS RAINFALL REGIONS AND BEGINNING AND END OF THE RAINY SEASON IN THE MIDDLE SAN FRANCISCO BASIN (BRAZIL)}

\footnotetext{
ABSTRACT

The water scarcity resulting from the poor Spatio-temporal distribution of rain generates great difficulties in water supply for the population of most of the semi-arid northeast and adjacent regions. In this way, knowledge about the rainfall regime of a Hydrographic Basin becomes
} 
essential, as a way to monitor droughts, in this case the Middle São Francisco Basin (BMSF) (Brazil). In this work it used daily precipitation data from 22 conventional meteorological stations of the Instituto Nacional de Meteorologia (INMET), located in the BMSF and adjacent areas, from 2001 to 2017 . For identification of rain patterns, Cluster analysis via Ward method was used for the discretization of homogeneous groups. Then, the seasonality of the basin was identified, as well as the beginning (IEC) and end (FEC) of rainfall via the Liebmann method. The BMSF rainfall regime was subdivided in to three homogeneous groups (G1, G2, and G3). In addition, these groups presented a similar seasonal pattern of rainfall, the dry season between the May and September months, and the rainy season between October and April months. The IEC and FEC started in mid-October and March, respectively.

Keywords: Hydrographic Basin. Rain. Cluster Analysis.

\section{INTRODUÇÃO}

A Bacia Hidrográfica do Médio São Francisco (BMSF) está situada na área do semiárido Nordestino Brasileiro, numa região altamente vulnerável do ponto de vista ambiental, em virtude dos solos arenosos e da vegetação que a recobre, além de recorrentes episódios de secas severas (MARENGO et al., 2016; CBHSF, 2018). A seca é um problema secular que afeta diversas regiões do mundo, no Brasil influencia principalmente o Nordeste Brasileiro (NEB), provocando perdas socioeconômicas, e impactando diretamente seus biomas (CUNHA et al., 2017; CORREIA FILHO et al., 2019). Os efeitos das secas afetam diferentes atividades humanas, tais como: agricultura, abastecimento d'água das cidades, produção industrial, pecuária e etc. (WILHITE, 2009; MAIA et al., 2015; CUNHA et al., 2017).

O entendimento das condições hídricas da BMSF é fundamental para a tomada de decisões políticas, principalmente em ações de mitigação dos impactos da seca (SILVA e CRUZ, 2017) e de perdas socioeconômicas na região. Sabe-se que a região do semiárido apresenta déficit hídrico em alguns meses do ano (abril a setembro), e que estas chuvas são distribuídas de forma irregular (KAYANO e ANDREOLI, 2009; SANTOS et al., 2017), ao qual resulta em eventos de secas ou enchentes em diferentes localidades (LYRA et al., 2017; CORREIA FILHO et al., 2018). Além disso, segundo Cruz, Ribeiro e Galizoni (2018) a evapotranspiração (ET) é quatro vezes maior que o total pluviométrico local. A determinação do início (IEC) e fim (FEC) da estação chuvosa pode melhorar o planejamento de diversas atividades humanas na região, tais como abastecimento d'água, indústria, pecuária e agricultura, sendo possível determinar as melhores áreas e períodos para o plantio. Existem diversas formas de se determinar essas datas, por exemplo, a partir de dados de chuva, radiação de onda longa (ROL), ou destas combinadas (MURAKAMI e NAZAWA, 1985; KOUSKY, 1988; SUGAHARA, 1991; MARENGO et al., 2001; LIEBMANN e MARENGO, 2001; LIEBMANN et al., 2007; VILLARON e FISCH, 2013; SANTOS e GARCIA, 2016). Como exemplo, no método proposto por Liebmann et al. (2007), os autores identificaram o IEC e FEC com base no "acumulado de anomalias" de chuva via dados observados e simulados, e constataram que no Sul da Amazônia o IEC ocorreu no dia 17 (18) de outubro para os dados observados (simulados), os quais correspondem às anomalias negativas de precipitação.

Anteriormente, Liebmann e Marengo (2001) definiram o IEC e FEC baseado nesse acumulado de anomalias de precipitação para a região do Sistema de Monção da América do Sul (SMAS), entre 19761997. Bombardi e Carvalho (2008) utilizaram metodologia similar à de Liebmann e Marengo (2001) com base em dados de precipitação estimados por satélite e medidas em superfície para o período de 1979 a 2004. Os autores verificaram a acurácia do método em caracterizar o IEC na Bacia Amazônica.Outro parâmetro importante para o estudo da seca é a distribuição espacial e identificação de regiões pluviométricas similares a partir da estatística multivariada, como por exemplo, a Análise de Cluster (AC). Este método consiste em caracterizar as variáveis de interesse, neste caso a chuva, de acordo com sua proximidade e padrões característicos e é muito utilizada para delimitação de regiões homogêneas, a partir de séries temporais de chuva (LYRA, OLIVEIRA-JÚNIOR e ZERI, 2014; OLIVEIRA-JÚNIOR et al., 2017). Melo Júnior et al. (2006) utilizaram AC para avaliar a chuva no leste do Estado de Minas Gerais. Os autores concluíram que o método de Ward (Ward, 1963) apresentou a melhor representação espacial para os grupos homogêneos. Guedes, Macedo e Sousa (2012) encontraram quatro grupos homogêneos de chuva para o Estado do Piauí, via método de Ward, enquanto que Gotardo et al. (2018), utilizaram a mesma metodologia, mas para o Estado de Santa 
Identificação das regiões pluviométricas homogêneas e início e fim da estação chuvosa na Bacia do Médio São Francisco (Brasil)
Laurizio Emanuel Ribeiro Alves Heliofábio Barros Gomes

Washington Luiz Félix Correia Filho José Francisco de Oliveira Júnior Luis Gustavo Gonçalves de Gonçalves

Dirceu Luis Herdies

Fabrício Daniel dos Santos Silva

Catarina e identificaram três grupos homogêneos, ao qual caracterizaram o padrão pluviométrico e a distribuição espacial da chuva em suas respectivas regiões.

Desta maneira, com o auxílio da $\mathrm{AC}$ aplicados às estações pluviométricas possibilitarão identificar o comportamento do início e fim do período chuvoso ao qual auxilie as tomadas de decisão quanto ao uso da água na BHSF, uma região que convive com restrição hídrica em decorrência da irregularidade das chuvas. Diante do exposto, o objetivo desta pesquisa consiste na identificação das regiões pluviométricas homogêneas e do início e fim da estação chuvosa na BMSF.

\section{MATERIAIS E MÉTODOS}

\section{ÁREA DE ESTUDO}

A BMSF está inserida numa das regiões mais secas do Brasil, o Semiárido Brasileiro recobre a parte norte e leste da bacia (Figura 1), além disso, apresenta o menor desenvolvimento socioeconômico das sub-bacias da Bacia Hidrográfica do Rio São Francisco (BHSF), ao qual cerca de 3,23 milhões de pessoas dependem da bacia (MMA, 2006). A BMSF tem uma área de $402.531 \mathrm{~km}^{2}$, ao qual $57 \%$ do seu total é urbanizada com uma densidade demográfica de $8 \mathrm{hab} / \mathrm{km}^{2}$. Os principais acidentes topográficos da BMSF são: Serra Geral de Goiás, Chapada da Diamantina, Chapadas das Mangabeiras e Serra da Tabatinga. Em relação aos biomas presentes na região são: Cerrado e Caatinga, mas predominada por pequenas matas de serra distribuídas ao longo da BMSF (ANA/GEF/PNUMA/OEA, 2003). A BMSF permeia 162 municípios, onde o Índice de Desenvolvimento Humano (IDH) varia entre 0,343 a 0,724 (PNUD, IPEA e FJP, 2013) e de acordo com a classificação de Köppen é clima "Aw", predominante tropical com inverno seco (ÁLVARES et al., 2013), com valores anuais de chuva entre $600 \mathrm{~mm}$ a $1.400 \mathrm{~mm}$, de evapotranspiração de $1.300 \mathrm{~mm}$ e de temperatura de $24^{\circ} \mathrm{C}$. O período chuvoso (seco) ocorre entre os meses de junho e agosto (novembro e janeiro), com $53 \%$ do total da chuva anual (CBHSF, 2018).

Figura 1 - Mapa de localização com as 22 estações meteorológicas convencionais do INMET e altitude (m) distribuídas ao longo da BMSF.

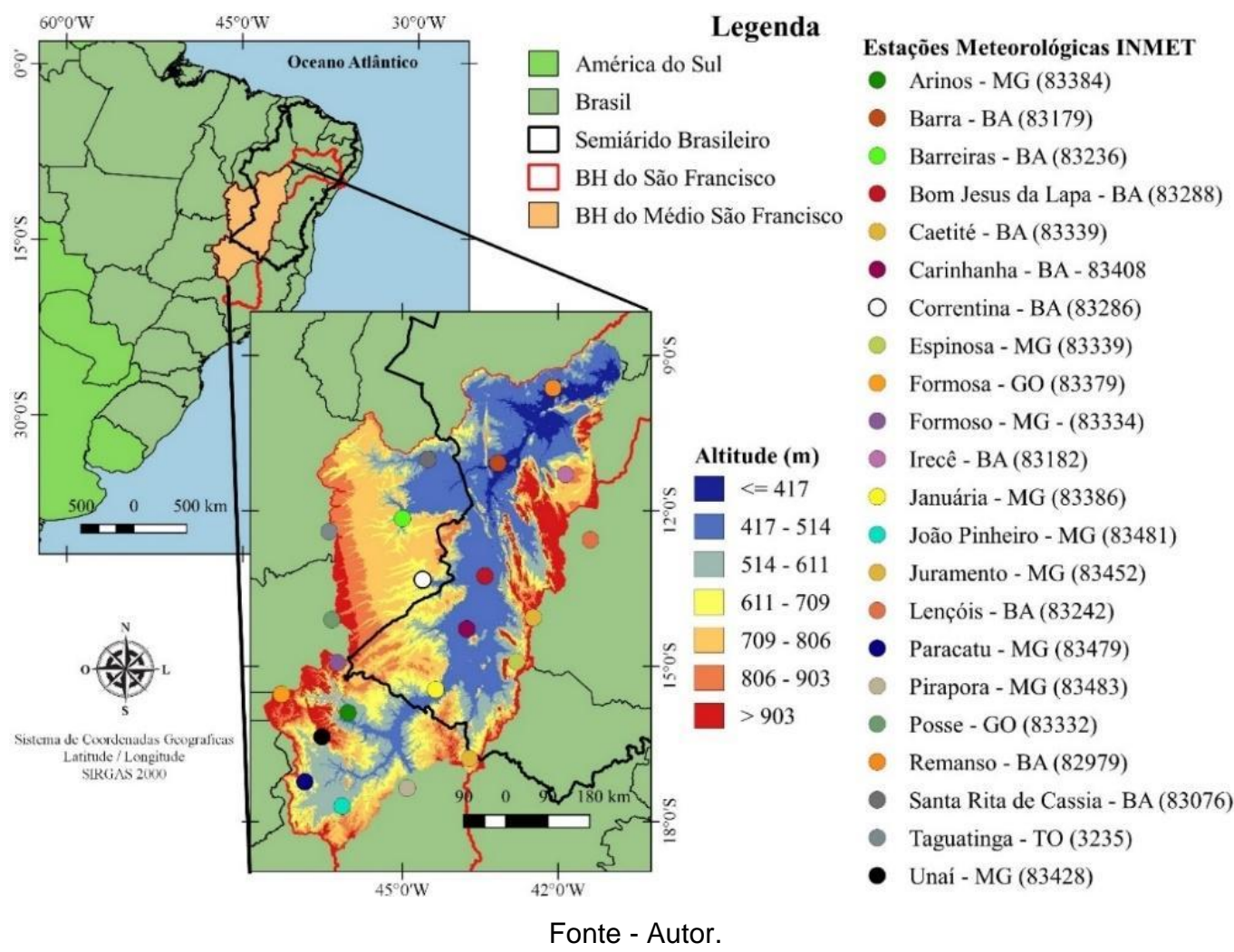


Identificação das regiões pluviométricas homogêneas e início e fim da estação chuvosa na Bacia do Médio São Francisco (Brasil)
Laurizio Emanuel Ribeiro Alves Heliofábio Barros Gomes

Washington Luiz Félix Correia Filho

José Francisco de Oliveira Júnior Luis Gustavo Gonçalves de Gonçalves Dirceu Luis Herdies

Fabrício Daniel dos Santos Silva

\section{AQUISIÇÃO E PROCESSAMENTO DOS DADOS DE CHUVA}

Para a análise da chuva da região foram utilizados dados diários de 22 estações meteorológicas convencionais (EMC) - (Figura 1), e as informações relativas às coordenadas geográficas encontramse descritas na Tabela 1. Os dados diários foram adquiridos via Banco de Dados Meteorológicos para Ensino e Pesquisa (BDMEP) do Instituto Nacional de Meteorologia (INMET) - (INMET, 2019), entre 2001 e 2017. O procedimento relativo à obtenção do percentual de Falhas (em \%) e preenchimento de falhas encontra-se com maiores detalhes em Alves e Gomes (2020), aos quais ambas as execuções foram realizadas pelo software livre R versão 3.4.1 (R DEVELOPMENT TEAM, 2017),

Tabela 1 - Informações das estações meteorológicas convencionais (código), com as coordenadas geográficas, identificador (ID), altitude (m) e percentual de falhas (\%).

\begin{tabular}{|c|c|c|c|c|c|}
\hline ID & Estação (Código) & Lat $\left({ }^{\circ}\right)$ & $\operatorname{Lon}\left({ }^{\circ}\right)$ & Altitude(m) & $\begin{array}{l}\text { Percentual de } \\
\text { Falhas (\%) }\end{array}$ \\
\hline 1 & Arinos - MG - 83384 & $-15,90$ & $-46,05$ & 519,00 & 0,1 \\
\hline 2 & Barra - BA - 83179 & $-11,08$ & $-43,16$ & 401,58 & 2,5 \\
\hline 3 & Barreiras - BA - 83236 & $-12,15$ & $-45,00$ & 439,29 & 5,5 \\
\hline 4 & Bom Jesus da Lapa - BA - 83288 & $-13,26$ & $-43,41$ & 439,96 & 0,2 \\
\hline 5 & Caetité - BA - 83339 & $-14,06$ & 42,48 & 882,47 & 0,1 \\
\hline 6 & Carinhanha - BA - 83408 & $-14,28$ & $-43,76$ & 450,18 & 4,6 \\
\hline 7 & Correntina - BA - 83286 & $-13,33$ & $-44,61$ & 549,47 & 11,0 \\
\hline 8 & Espinosa - MG - 83338 & $-14,91$ & $-42,80$ & 569,64 & 10,4 \\
\hline 9 & Formosa - GO - 83379 & $-15,53$ & $-47,33$ & 935,19 & 0,0 \\
\hline 10 & Formoso - MG - 83334 & $-14,93$ & $-46,25$ & 840,00 & 7,8 \\
\hline 11 & Irecê - BA - 83182 & $-11,30$ & $-41,86$ & 747,16 & 2,2 \\
\hline 12 & Januária - MG - 83386 & $-15,45$ & $-44,36$ & 473,71 & 0,9 \\
\hline 13 & João Pinheiro - MG - 83481 & $-17,70$ & $-46,16$ & 760,36 & 4,6 \\
\hline 14 & Juramento - MG - 83452 & $-16,78$ & $-43,71$ & 650,00 & 2,0 \\
\hline 15 & Lençóis - BA - 83242 & $-12,56$ & $-41,38$ & 438,74 & 0,1 \\
\hline 16 & Paracatu - MG - 83479 & $-17,23$ & $-46,88$ & 712,00 & 0,4 \\
\hline 17 & Pirapora - MG - 83483 & $-17,35$ & $-44,91$ & 505,24 & 4,5 \\
\hline 18 & Posse - GO - 83332 & $-14,10$ & $-46,36$ & 825,64 & 0,0 \\
\hline 19 & Remanso - BA - 82979 & $-9,63$ & $-44,51$ & 500,51 & 0,3 \\
\hline 20 & Santa Rita de Cassia - BA - 83076 & $-11,01$ & $-44,51$ & 450,30 & 1,9 \\
\hline 21 & Taguatinga - TO - 83235 & $-12,40$ & $-46,41$ & 603,59 & 0,1 \\
\hline 22 & Unaí - MG - 83428 & $-16,36$ & $-46,55$ & 460,00 & 0,0 \\
\hline
\end{tabular}

Fonte - INMET (2019). 
Identificação das regiões pluviométricas homogêneas e início e fim da estação chuvosa na Bacia do Médio São Francisco (Brasil)
Laurizio Emanuel Ribeiro Alves Heliofábio Barros Gomes Washington Luiz Félix Correia Filho José Francisco de Oliveira Júnior Luis Gustavo Gonçalves de Gonçalves Dirceu Luis Herdies

Fabrício Daniel dos Santos Silva

\section{ANÁLISE DE CLUSTER}

A análise de Cluster (AC) é um método de análise multivariada que busca identificar uma estrutura classificatória ou de reconhecimento a partir de um conjunto de indivíduos, baseado na avaliação da homogeneidade dentro dos grupos e/ou heterogeneidade entre os grupos (CORREIA FILHO e ARAGÃO, 2014; RIBEIRO FILHO, 2016). O procedimento de AC hierárquico foi empregado na série temporal pluviométrica mensal de chuva para as 22 EMC no software $R$ versão 3.4 .1 ( $R$ DEVELOPMENT TEAM, 2017). Desse modo, definiram-se os respectivos números de grupos a partir do dendrograma baseado no método de Ward via medida de dissimilaridade e distância euclidiana. De acordo com Ribeiro Filho (2016), o cálculo da distância euclidiana $\left(d_{x} y\right)$ entre dois elementos $X=\left[X_{1}, X_{2}\right.$, $\left.\ldots, X_{n}\right]$ e $Y=\left[Y_{1}, Y_{2}, \ldots, Y_{n}\right]$ é definido pela Equação 1.

$$
d_{x} y=\sqrt{\left(X_{1}-Y_{1}\right)^{2}+\left(X_{2}-Y_{2}\right)^{2}+\ldots+\left(X_{n}-Y_{n}\right)^{2}}=\sqrt{\sum_{i=1}^{N}\left(X_{1}-Y_{1}\right)^{2}}
$$

No método de Ward, também conhecido como "Variação Mínima", a distância entre os grupos é dada pela soma dos quadrados dos agrupamentos, maximizando a homogeneidade dentro dos grupos e a heterogeneidade fora deles (MINGOTI, 2007). Este método é muito utilizado em dados de precipitação (LYRA, OLIVEIRA-JÙNIOR e ZERI, 2014; OLIVEIRA-JÚNIOR et al., 2017). Para avaliar a qualidade da escolha do método e do número dos grupos, aplicou-se o Coeficiente de Correlação Cofenética (CCC), dado pela Equação 2.

$$
C C C=\frac{\sum_{i=1}^{n-1} \sum_{j=i+1}^{n}\left(c_{i j}-c m\right)\left(d_{i j}-d m\right)}{\sqrt{\sum_{i=1}^{n-1} \sum_{j=i+1}^{n}\left(c_{i j}-c m\right)^{2}} \sqrt{\sum_{i=1}^{n-1} \sum_{j=i+1}^{n}\left(d_{i j}-d m\right)^{2}}}
$$

Em que, $c_{i j}$ é o valor de dissimilaridade entre as amostras, obtidas via matriz cofenética; $d_{i j}$ é o valor de dissimilaridade entre amostras, obtidas a partir da matriz de dissimilaridade. $O \mathrm{~cm}_{\mathrm{m}}$ e $\circ \mathrm{dm}_{\mathrm{m}}$ são obtidos através das Equações 3 e 4, respectivamente.

$$
\begin{gathered}
c m=\frac{2}{n(n-1)} \sum_{i=1}^{n-1} \sum_{j=i+1}^{n} c_{i j} \\
d m=\frac{2}{n(n-1)} \sum_{i=1}^{n-1} \sum_{j=i+1}^{n} d_{i j}
\end{gathered}
$$

De acordo com Carvalho (2018), o CCC equivale à correlação de Pearson entre as matrizes de dissimilaridade original e a gerada após a construção do dendrograma. Desse modo, quanto mais se aproxima do 1 , indica uma maior homogeneidade do grupo e, consequentemente, melhor qualidade do agrupamento.

\section{MÉTODO DE DETECÇÃO DO INÍCIO E FIM DA ESTAÇÃO CHUVOSA - MÉTODO LIEBMANN}

O método de detecção do início (IEC) e fim (FEC) da estação chuvosa desenvolvido por Liebmann et al. (2007) utiliza dados diários de chuva, e se baseia no "acumulado de anomalias" obtido por cada estação meteorológica ou ponto de grade. Nesse método, o IEC (FEC) é definido pelo mínimo (máximo) da curva (Figura 2), isto é, as inflexões apresentadas no gráfico do acumulado de anomalias, calculado pela Equação 5. 
Identificação das regiões pluviométricas homogêneas e início e fim da estação chuvosa na Bacia do Médio São Francisco (Brasil)
Laurizio Emanuel Ribeiro Alves Heliofábio Barros Gomes Washington Luiz Félix Correia Filho José Francisco de Oliveira Júnior Luis Gustavo Gonçalves de Gonçalves Dirceu Luis Herdies Fabrício Daniel dos Santos Silva

$$
A(\text { dia })=\sum_{n=1}^{\text {dia }}\left[R(n)-R_{m}\right]
$$

Em que, $A$ (dia) é o acumulado de anomalias pluviométricas, $R(n)$ é a precipitação diária e $R_{m}$ é a média anual climatológica diária de precipitação, ou seja, o somatório da anomalia de chuva do dia anterior e o dia vigente. Liebmann et al. (2007) recomenda que o cálculo das anomalias pode ser iniciado em qualquer momento do ano, mas o ideal é que o acumulado de anomalias seja iniciado 10 dias antes do mês mais seco.

Figura 2 - Determinação do IEC e FEC no gráfico de acumulado de anomalias de chuva (mm). Linha continua azul - dados observados; linha tracejada vermelha - suavização dos dados observados; dados ilustrativos).

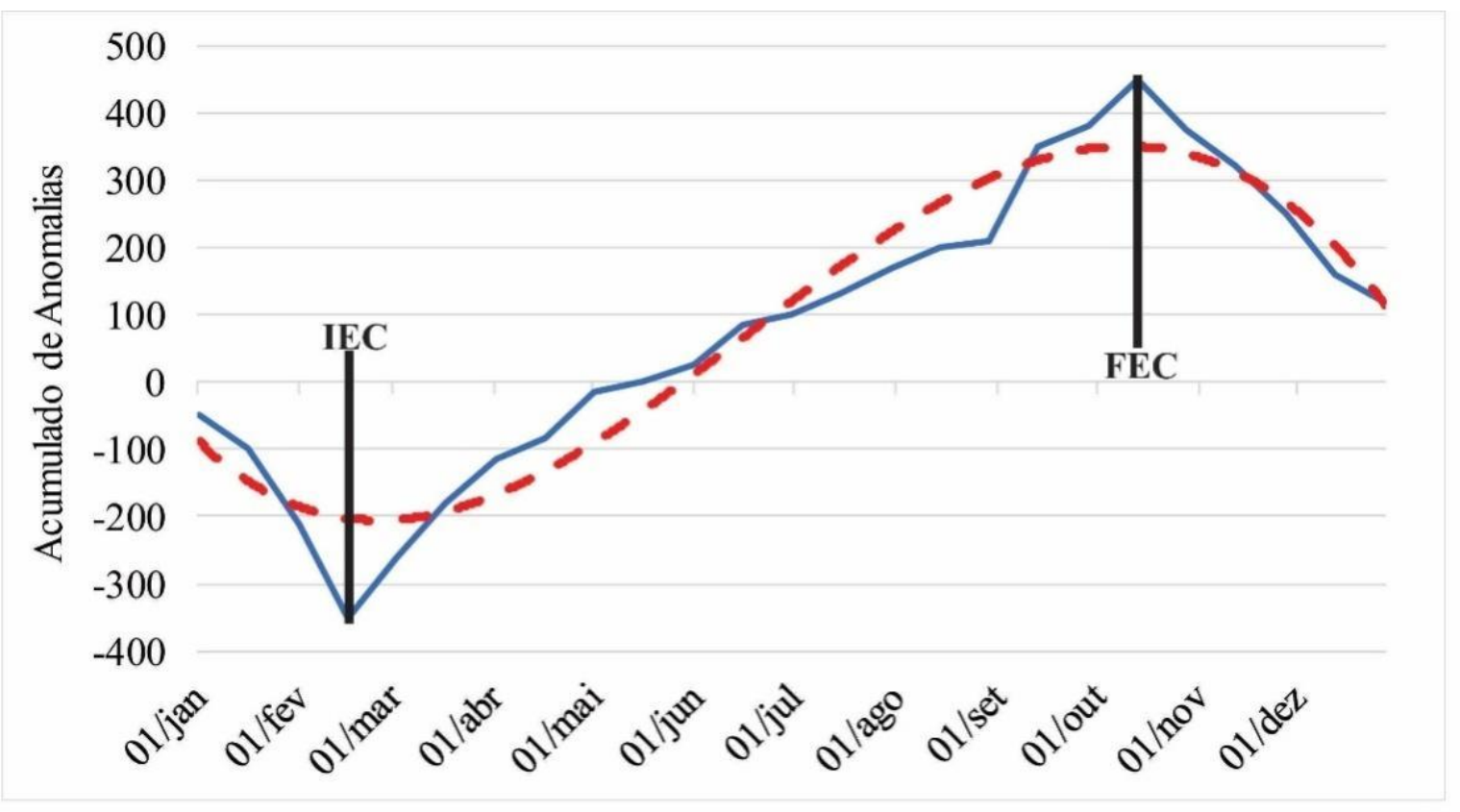

\section{RESULTADOS E DISCUSSÃO}

\section{ANÁLISE DE CLUSTER}

A partir da aplicação da AC nos dados diários de chuva sobre a BMSF foram identificados três grupos pluviométricos homogêneos (G1, G2 e G3), ilustrada na Figura 3. A quantidade de grupos se mostra adequada de acordo com a CCC que apresentou o valor de 0,729. De acordo com Rohlf (1962) e Carvalho (2018), quando os valores de CCC são superiores a 0,7 , indica que os dados foram agrupados adequadamente, ou seja, os três grupos da BMSF são congruentes, com distância euclidiana inferior a 400. O grupo G1 é formado por seis estações (menor grupo), enquanto os grupos G2 e G3 foram formados por oito estações cada (maiores grupos), ao qual é exibida a composição dos grupos na Tabela 2.

A Figura 4 mostra a distribuição espacial das estações pertencentes aos diferentes grupos, com diferentes cores associadas: Grupo 1 (G1, preto), Grupo 2 (G2, vermelho) e Grupo 3 (G3, verde). No grupo G1, o Total Pluviométrico (TP) varia entre $923,9 \pm 117,7 \mathrm{~mm}$ a $1225,7 \pm 117,7 \mathrm{~mm}$, com MM \pm DP de $121,3 \pm 105,4 \mathrm{~mm}$, e representa cerca de $36 \%$ da chuva da BMSF. Já no grupo G2, o TP oscilou entre $1360,7 \pm 87,3 \mathrm{~mm}$ a $1611,5 \pm 87,3 \mathrm{~mm}$, com MM \pm DP de $86,7 \pm 80,5 \mathrm{~mm}$ e representa $38 \%$ do total 
Identificação das regiões pluviométricas homogêneas e início e fim da estação chuvosa na Bacia do Médio São Francisco (Brasil)
Laurizio Emanuel Ribeiro Alves Heliofábio Barros Gomes

Washington Luiz Félix Correia Filho José Francisco de Oliveira Júnior Luis Gustavo Gonçalves de Gonçalves Dirceu Luis Herdies

Fabrício Daniel dos Santos Silva

da BMSF. Este grupo se distribui sobre a região sudeste, central, e noroeste da BMSF, mas está mais próxima do Semiárido Brasileiro, apresenta TP superiores ao grupo G1. O grupo G3 é o mais seco, com TP entre $609 \pm 144,9 \mathrm{~mm}$ a $1068,7 \pm 144,9 \mathrm{~mm}$ e MM $\pm \mathrm{DP}$ de $62,9 \pm 55,4 \mathrm{~mm}$, e contribui com $26 \%$ do TP da BMSF.

As estações do G2 possuem os maiores TP, estão situadas numa região de vale, e os padrões de vento e umidade local contribuem para formação de chuva orográfica. Reboita et al. (2014) relatam que regiões de vale circundada por áreas elevadas topograficamente, em regra, com um escoamento que se conduz do vale para a montanha durante o dia, esse escoamento do vento é conhecido como ventos anabáticos e, quando com umidade suficiente, podem causar chuva.

Figura 3 - Dendrograma com os três grupos homogêneos, obtidas a partir dos dados diários de chuva das 22 EMC situadas na BMSF.

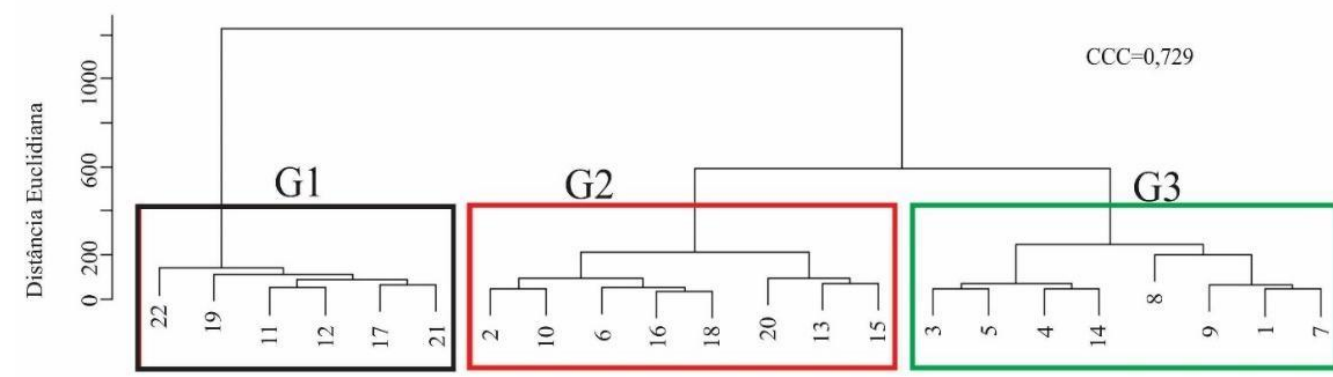

dist(var)

hclust(*,ward.D)

Tabela 2 - Composição dos três grupos homogêneos baseados nas 22 EMC, a partir dos dados de chuva situadas na BMSF. Os valores que se encontram dentro de cada parêntese correspondem à Média Mensal (MM) \pm Desvio Padrão (DP) de cada grupo homogêneo.

\begin{tabular}{ll}
\hline Grupos (MM \pm DP) & EMC \\
\hline G1 $(81,1 \pm 72,3)$ & Taguatinga - TO, Formosa - GO, João Pinheiro - MG, Paracatu - MG, Posse - \\
& GO, Unaí - MG \\
\hline G2 $(91,0 \pm 80,5)$ & $\begin{array}{l}\text { Arinos - MG, Barreiras - BA, Correntina - BA, Formoso - MG, Januária - MG, } \\
\text { Juramento - MG, Pirapora - MG, Santa Rita de Cássia - BA }\end{array}$ \\
\hline G3 $(75,7 \pm 55,4)$ & Remanso - BA, Barra - BA, Bom Jesus da Lapa - BA, Caetité - BA, Carinhanha \\
& - MG, Espinosa - MG, Irecê - BA, Lençóis - BA \\
\hline
\end{tabular}

\section{ANÁLISE EXPLORATÓRIA DA CHUVA NA BMSF}

Após o diagnóstico da distribuição e representação espacial dos grupos homogêneos, avaliou-se o comportamento sazonal da chuva de cada um dos três grupos, a partir de gráficos boxplot (Figura 5) e da estatística descritiva (Tabela 3). Em geral, os grupos homogêneos exibem que o período chuvoso (seco) ocorre entre os meses de outubro a abril (maio a setembro).

Os maiores TP ocorrem durante o período chuvoso, ao qual estão associados aos sistemas meteorológicos em multiescala, por exemplo, Vórtices Ciclônicos de Altos Níveis (VCAN), Zona de Convergência Intertropical (ZCIT), Linhas de Instabilidade (LI), Complexos Convectivos de Mesoescala (CCM), Zona de Convergência do Atlântico Sul (ZCAS), associados aos fatores regiões ou locais (MOLION e BERNARDO, 2002; SILVA e BRITO, 2008; COUTINHO, 2008; REBOITA et al., 2010; COUTINHO, GAN e RAO, 2010; LYRA, OLIVEIRA-JÚNIOR e ZERI, 2014; LYRA, 2018). 
Identificação das regiões pluviométricas homogêneas e início e fim da estação chuvosa na Bacia do Médio São Francisco (Brasil)
Laurizio Emanuel Ribeiro Alves Heliofábio Barros Gomes

Washington Luiz Félix Correia Filho José Francisco de Oliveira Júnior Luis Gustavo Gonçalves de Gonçalves Dirceu Luis Herdies

Fabrício Daniel dos Santos Silva

Durante o período seco, a atuação destes sistemas meteorológicos é reduzida de maneira significativa e a maior parte deles não ocorre no período, resultado da forte atuação da Alta Subtropical do Atlântico Sul (ASAS) que contribui para a redução dos totais pluviométricos da região (DEGOLA, 2013). Vale destacar que o modo de variabilidade climática EI Niño-Oscilação Sul (ENOS) foi marcante para a redução da chuva na região, pois de acordo com Marengo, Cunha e Alves (2016) o evento forte de EI Niño de 2015-2016 intensificou a seca que ocorria no semiárido nordestino. Correia Filho et al. (2018) relataram que, apesar da ocorrência de um evento de La Niña forte em 2012, que em média é uma condicionante para o aumento das chuvas no semiárido, as condições desfavoráveis associadas ao dipolo do atlântico com forte sinal positivo daquele ano reduziu a chuva anual sobre a Caatinga em mais de $45 \%$, e nesse período foi declarado estado de emergência pública (MARENGO et al., 2018).

Figura 4 - Distribuição espacial dos grupos homogêneos (G1, G2 e G3) na região BMSF (Brasil).

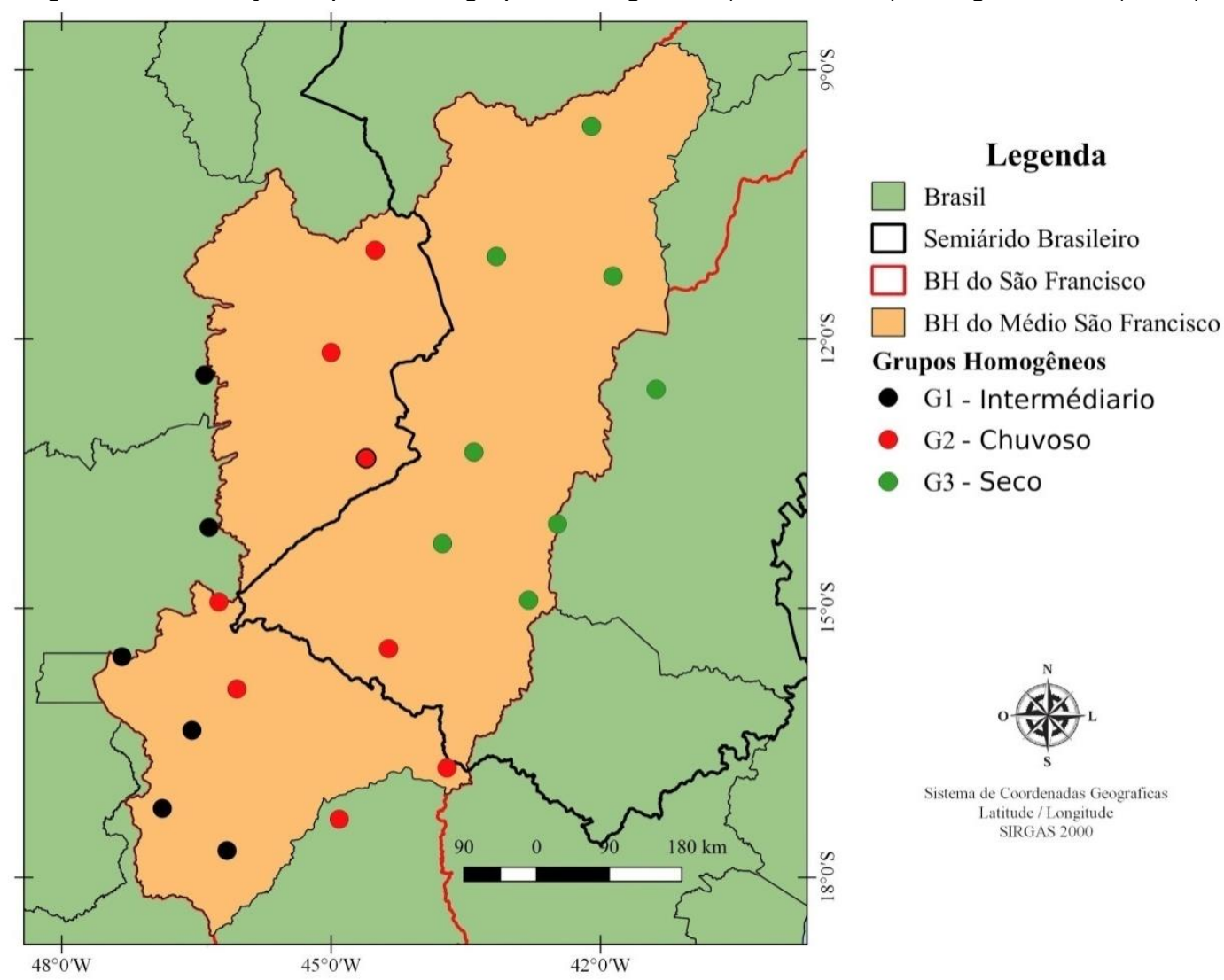

O padrão sazonal das chuvas dos três grupos homogêneos (Figura 5) apresenta a ocorrência de outliers. Além disso, o grupo G3 apresenta um outlier no mês de dezembro (Figura 6c) relativo a um extremo de mínima mensal, possivelmente ocasionado por padrões anômalos que resultaram na redução significativa de chuvas neste mês. Ademais, durante o período chuvoso (entre os meses de novembro e março) há a presença de poucos episódios de outliers nos três grupos homogêneos, associados a ocorrência de eventos extremos de chuva, com índices pluviométricos superiores a 275 $\mathrm{mm} / \mathrm{mês}$. No período seco (entre os meses de maio e setembro), também se constata a presença de outliers com valores entre $20-55 \mathrm{~mm} / \mathrm{mês}$. No geral, na BMSF ocorreram 15 outliers no período chuvoso e a mesma quantidade de outliers no período seco. 
Identificação das regiões pluviométricas homogêneas e início e fim da estação chuvosa na Bacia do Médio São Francisco (Brasil)
Laurizio Emanuel Ribeiro Alves Heliofábio Barros Gomes

Washington Luiz Félix Correia Filho José Francisco de Oliveira Júnior Luis Gustavo Gonçalves de Gonçalves

Dirceu Luis Herdies

Fabrício Daniel dos Santos Silva

Tabela 3 - Estatística descritiva (mínimo, 1ำ quartil, mediana, média, 3ำ quartil e máximo) dos três grupos homogêneos (G1, G2 e G3) obtidos pela técnica de $A A$.

\begin{tabular}{llll}
\hline Parâmetros & G1 & G2 & G3 \\
\hline Mínimo & 0,0 & 1,3 & 0,1 \\
\hline $1^{\circ}$ Quartil & 3,7 & 10,1 & 4,2 \\
\hline Mediana & 42,8 & 54,7 & 40,5 \\
\hline Média & 81,1 & 91,0 & 75,7 \\
\hline 30 Quartil & 134,7 & 158,2 & 132,7 \\
\hline Máxima & 355,5 & 502,0 & 431,9
\end{tabular}

Em janeiro de 2016 ocorreu um outlier no grupo G2 (Figura 6b), resultante de sucessivos episódios de extremos de chuva ao qual superaram a média climatológica do período, de 166,2 mm para $502 \mathrm{~mm}$ (um acréscimo de 202,4\% na média do mês), descritos na Tabela 3 . Este alto índice de chuva causou alagamentos na região. Os casos de alagamentos foram noticiados pela REDEBAHIA do Grupo Globo com a seguinte manchete: "Forte chuva causa alagamentos e prejuízos em cidades da Bahia", na reportagem relatam que "Em Barreiras, na região oeste, já choveu quatro vezes mais que o previsto para todo o mês de janeiro" (GRUPO GLOBO - BA, 2016).

Figura 5 - Boxplot mensal da chuva (mm) dos grupos homogêneos de chuva G1 (a), G2 (b) e G3 (c) para a BMSF.

(d)

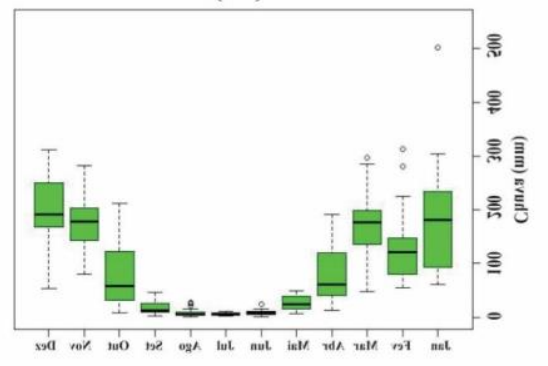

( s)

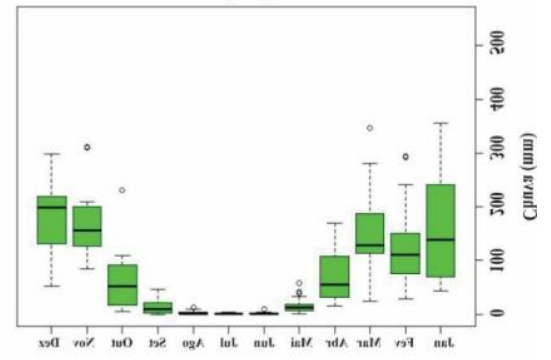

( э)

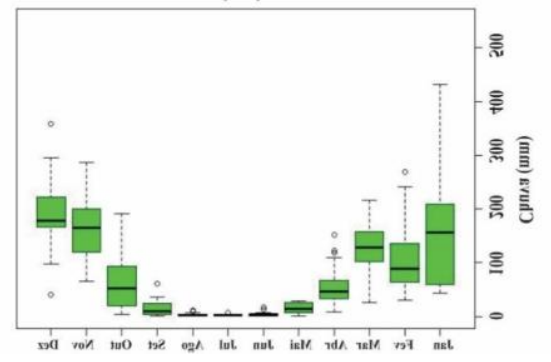

Estes eventos estão relacionados com a atuação da ZCAS sobre a região Sudeste do Brasil e com a formação de um Ciclone Tropical posicionado no Oceano Atlântico próximo aos estados do Rio de Janeiro, Espírito Santo e Bahia, conforme constatado por Correia Filho et al. (2018). A associação destes eventos no sul da BA contribuiu para os volumes expressivos de chuva ao longo do Semiárido, especialmente na região leste e noroeste da Bahia, pois com valores entre 400-500 mm.

Os outliers registrados no período seco se devem a média baixa de chuva na região, que não ultrapassa $10 \mathrm{~mm} / \mathrm{mês}$. Devido a essas características, valores que superem esta média já são considerados 
Identificação das regiões pluviométricas homogêneas e início e fim da estação chuvosa na Bacia do Médio São Francisco (Brasil)
Laurizio Emanuel Ribeiro Alves Heliofábio Barros Gomes

Washington Luiz Félix Correia Filho José Francisco de Oliveira Júnior Luis Gustavo Gonçalves de Gonçalves

Dirceu Luis Herdies

Fabrício Daniel dos Santos Silva

outliers. No entanto, no ano de 2012, a presença do outlier está associada à seca extrema que afetou todo o NEB, entre os anos de 2012 e 2015, conforme constatado por Marengo, Torres e Alves (2017) e Correia Filho et al. (2018). Este período de seca prolongada causou enormes perdas socioeconômicas não apenas para a Região de BMSF, como para todo o NEB. O prejuízo decorrente desta seca foi de aproximadamente $\mathrm{R} \$ 103,5$ bilhões e afetou cerca de 33,4 milhões de pessoas (CORREIA FILHO et al., 2018), principalmente no Estado da Bahia que decretou situação de emergência em mais de 220 municípios naquele ano (ARAÚJJO, 2012).

Nota-se que o mês de janeiro apresenta maior amplitude em seus mínimos e máximos de chuva, enquanto que o mês de dezembro apresenta a maior média mensal, para todos os grupos. Já no período seco (entre os meses de maio e setembro) os TP não ultrapassam $60 \mathrm{~mm} / \mathrm{mês}$, enquanto que no período chuvoso a chuva facilmente excede os $100 \mathrm{~mm} / \mathrm{mês}$.

\section{IDENTIFICAÇÃO DO INÍCIO E FIM DO PERÍODO CHUVOSO NA BMSF}

Para os grupos G1, G2 e G3 (Figura 6), o IEC (FEC) corresponde às datas 04/10 (16/03), 06/10 (20/03) e 05/10 (15/03), respectivamente. De acordo com os resultados, os meses de março e início de outubro correspondem ao fim e início da estação chuvosa na BMSF em 2001. Para o ano de 2017, o IEC (FEC) ocorreu em 27/10 (02/04), 27/10 (04/04), 29/10 (05/04). Neste ano, o fim e o início da estação chuvosa ocorreram nos meses de abril e final de outubro. No geral, entre 2001 a 2017, o IEC (FEC) para os três grupos coincidem às datas 14/10 (20/03), 16/10 (26/03), 17/10 (30/03).

Figura 6 - Acumulado de Anomalias de chuva ( $\mathrm{mm}$ ) para a BMSF dos grupos homogêneos G1 (linha de cor preta), G2 (linha de cor vermelha) e G3 (linha de cor verde), para os anos de 2001 (a) e 2017 (b), respectivamente.
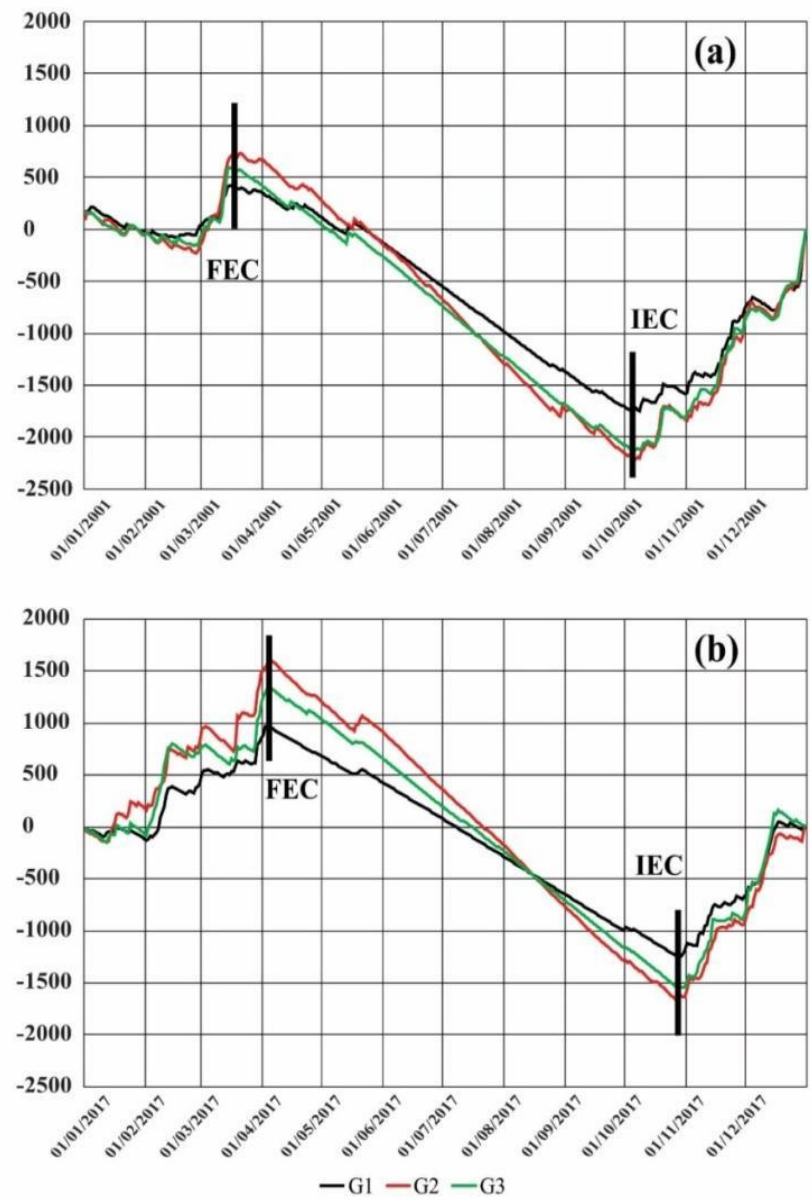
Identificação das regiões pluviométricas homogêneas e início e fim da estação chuvosa na Bacia do Médio São Francisco (Brasil)
Laurizio Emanuel Ribeiro Alves Heliofábio Barros Gomes

Washington Luiz Félix Correia Filho José Francisco de Oliveira Júnior Luis Gustavo Gonçalves de Gonçalves Dirceu Luis Herdies

Fabrício Daniel dos Santos Silva

Para o ano de 2001, em média, o IEC ocorre no dia 278 ( $\pm 1,00$ dia) e o FEC ocorre no dia $76( \pm 2,65$ dias), ao qual condizem aos meses de setembro e março, respectivamente. No ano de 2017, o IEC foi iniciado no dia 301 ( $\pm 1,53 \mathrm{dia}$ ) e FEC em 94 ( $\pm 1,15 \mathrm{dia}$ ), os quais correspondem aos meses de outubro e abril, respectivamente. Resultados similares para a determinação do IEC foram encontrados por Alves et al. (2005), que determinaram que o início da estação chuvosa na região Sudeste do Brasil ocorre entre os dias 08-12 de outubro. Eles relacionaram a antecipação (atraso) do IEC a anomalias positivas (negativa) de Temperatura da Superfície do Mar (TSM) do Atlântico. Bombardi e Carvalho (2008) encontraram o IEC entre as pêntadas 56 a 60 na região Centro-Oeste e Sudeste do Brasil, que correspondem de setembro a outubro. Santos e Garcia (2016) avaliaram o início e fim da estação chuvosa no Estado de Minas Gerais e verificaram que o IEC para o período de 2001-2002 foi iniciado na pêntada 55, que corresponde ao mês de setembro. Ademais o FEC ocorreu na pêntada 13, que corresponde ao mês de março.

$\mathrm{Na}$ região central da BMSF, correspondente ao grupo G2 (Figura 6), verifica-se os maiores totais de anomalias de chuva. Esse padrão já era esperado, já que a região é uma área de incursão de frentes, que geralmente fica estacionária sobre essas latitudes (FERREIRA e MELLO, 2005). Outros fenômenos sinóticos importantes que causam chuva forte na região são: VCAN, ZCAS e atuação de CCMs, além da atuação de convergência devido ao efeito topográfico (RAO, LIMA e FRANCHITO, 1993; SILVA e BRITO, 2008; COUTINHO, 2008; COUTINHO, GAN e RAO, 2010), já que a área central da BMSF se encontra numa região de vale, contribuindo para o aumento da chuva local.

\section{CONSIDERAÇÕES FINAIS}

O regime pluviométrico da BMSF é bem definido, com um período seco entre os meses de abril e setembro, e chuvoso entre os meses de outubro e março. A Análise de Cluster via método de Ward identificou três grupos homogêneos, validado pelo CCC com o valor de 0,729. O grupo G1 localizado no leste e sul da BMSF, o grupo G2 é localizado entre o sudeste, centro e noroeste e o grupo G3 é composto basicamente por estações situadas na porção da BMSF localizada no semiárido brasileiro. Tais grupos têm variabilidade espaço-temporal da chuva em função da atuação de sistemas meteorológicos multiescala e, ainda a influência de fatores fisiográficos, por exemplo, topografia complexa. O método de Liebmann et al. (2007) se mostrou aplicável para a BMSF, e apontou que o início da estação chuvosa para a BMSF ocorre no mês de outubro, enquanto o fim do período em março, com pequenas diferenças entre os grupos.

\section{REFERÊNCIAS BIBLIOGRÁFICAS}

ALVARES, C.A.; STAPE, J. L.; SENTELHAS, P. C.; DE MORAES GONÇALVES, J. L.; SPAROVEK, G. Köppen's climate classification map for Brazil. Meteorologische Zeitschrift, v. 22 n. 6, p.711-728. 2013. ISSN 1610-1227. https://doi.org/10.1127/0941-2948/2013/0507

ALVES, L. M.; MARENGO, J. A.; JÚNIOR, H. C.; CASTRO, C. Início da estação chuvosa na região sudeste do Brasil: Parte 1 - Estudos observacionais. Revista Brasileira de Meteorologia, v. 20, n. 3, p. 385-394, 2005. ISSN 1982-4351. https://doi.org/10.11137/2020 1199206

ALVES, L.E. R.; GOMES, H. B. Validação da Imputação Múltipla via PMM para dados pluviométricos na Bacia do Médio São Francisco. Anuário do Instituto de Geociências, Rio de Janeiro - RJ, v. 43, n. 1, p., 2020.

ANA - Agência Nacional das Águas. GEF - Fundo para o Meio Ambiente Mundial. PNUMA - Programa das Nações Unidas para o Meio Ambiente. OEA - Organização dos Estados Americanos. Projeto de Gerenciamento Integrado das Atividades Desenvolvidas em Terra na Bacia do São Francisco, Sub-projeto 4.5.B - Programa de ações estratégicas para o gerenciamento integrado da Bacia do Rio São Francisco e da sua zona costeira - PAE: Brasília: 2003. 243 p.

ARAÚJO, G. Seca na Bahia é a maior dos últimos 47 anos, diz secretário da Casa Civil. G1, 05, maio 2012. Disponível em: <http://glo.bo//Zh0yU>. Acessado em: 29 de out. 2019. 
Identificação das regiões pluviométricas homogêneas e início e fim da estação chuvosa na Bacia do Médio São Francisco (Brasil)
Laurizio Emanuel Ribeiro Alves Heliofábio Barros Gomes

Washington Luiz Félix Correia Filho José Francisco de Oliveira Júnior Luis Gustavo Gonçalves de Gonçalves Dirceu Luis Herdies

Fabrício Daniel dos Santos Silva

BOMBARDI, R. J.; CARVALHO, L. M. V. Variabilidade do regime de monções sobre o Brasil: O clima presente e projeções para um cenário com 2xCO2 usando o modelo MIROC. Revista Brasileira de Meteorologia, v. 23, n. 1, p. 58-72, 2008. ISSN 1982-4351. https://doi.org/10.1590/S0102$\underline{77862008000100007}$

CARVALHO, P. R. Estudo comparativo dos algoritmos hierárquicos de análise de agrupamento em resultados experimentais. 2018. 142 f. Dissertação (Mestre em Ciências) - Universidade de São Paulo, São Paulo, 2018.

CBHSF - Comitê da Bacia Hidrográfica do Rio São Francisco. CBH Afluentes do Médio São Francisco (SF9) - Minas Gerais. Disponível em: < http://cbhsaofrancisco.org.br/2017/comites-deafluentes/cbh-afluentes-mineiros-do-medio-sao-francisco-minas-gerais/>. Acessado em 08 de ago. 2018.

CORREIA FILHO, W. L. F.; OLIVEIRA JÚNIOR, J. F. de; SANTIAGO, D. B.; TERASSI, P. M. B.; TEODORO, P. E.; GOIS, G.; BLANCO, C. J. C.; SOUZA, P. H. A.; COSTA, M.; SANTOS, P. J. Rainfall variability in the Brazilian northeast biomes and their interactions with meteorological systems and ENSO via CHELSA product. Big Earth Data, v. 3, p. 315-337, 2019. https://doi.org/10.1080/20964471.2019.1692298. https://doi.org/10.1080/20964471.2019.1692298

CORREIA FILHO, W. L. F.; SANTOS, T. V.; DIOGO, D. M.; AMORIM, R. F. C. Diagnóstico da Precipitação e EVI em dois eventos de seca no Nordeste do Brasil. Revista do Departamento de Geografia, v.35, p. 102-112, 2018. https://doi.org/10.11606/rdg.v35i0.140068. https://doi.org/10.11606/rdg.v35i0.140068

CORREIA FILHO, W. L. F.; ARAGÃO, M. R. S. Padrões temporais do vento à superfície em mesorregiões do estado da Bahia. Ciência e Natura, v. 36, p. 402-414, 2014.https://doi.org/10.5902/2179460X13162. https://doi.org/10.5902/2179460X13162

COUTINHO, M. D. L. Método objetivo de identificação dos Vórtices Ciclônicos de Altos Níveis na região tropical sul: Validação e climatologia. 2008. 119f. Dissertação (Mestrado em Meteorologia) Instituto Nacional de Pesquisas Espaciais, São José dos Campos, 2008.

COUTINHO, M. D. L.; GAN, M. A.; RAO, V. B. Método Objetivo de Identificação dos Vórtices Ciclônicos e Altos Níveis na Região Tropical Sul: Validação. Revista Brasileira de Meteorologia, v. 25, n.3, p. 311-323, 2010. ISSN 1982-4351. https://doi.org/10.1590/S0102-77862010000300003

CRUZ, G. C.; RIBEIRO, E. M.; GALIZONI, F. M. SEMIÁRIDO, SECA E "GERAIS" DO NORTE DE MINAS: uma revisão da bibliografia sobre o Alto-Médio São Francisco. CAMPO-TERRITÓRIO: revista de geografia agrária, v.13, n. 31, p. 29-56, 2018. https://doi.org/10.14393/RCT133102

CUNHA, A. P. M. A. et al. Avaliação de indicador para o monitoramento dos impactos da seca em áreas de pastagens no semiárido do Brasil. Revista Brasileira de Cartografia. v. 6, n. 1, p. 89-106, 2017. ISSN 1808-0936.

DEGOLA, T. S. D. Impactos e variabilidade do Anticiclone Subtropical do Atlântico Sul sobre o Brasil no clima presente e em cenários futuros. 2013. 112f. Dissertação (Mestrado em Meteorologia) - Universidade de São Paulo, São Paulo, 2013.

FERREIRA, A. G.; MELLO, N. G. S. Principais sistemas atmosféricos atuantes sobre a região nordeste do Brasil e a influência dos oceanos Pacífico e Atlântico no clima da região. Revista Brasileira de Climatologia, v. 1, n. 1, p. 15-28, 2005. ISSN 2237-8642. https://doi.org/10.5380/abclima.v1i1.25215

GOTARDO, R., PIAZZA, G., TORRES, E., SEVERO, D., KAUFMAN, V. Distribuição espacial e temporal das chuvas no Estado de Santa Catarina. Geosul, Florianópolis - SC, v. 33, n. 67, p. 253-276, 2018. https://doi.org/10.5007/2177-5230.2018v33n67p253

GRUPO GLOBO - BA. Forte chuva causa alagamentos e prejuízos em cidades da Bahia. G1, 28, janeiro 2016. Disponível em: < http://g1.globo.com/bahia/noticia/2016/01/forte-chuva-causaalagamentos-e-prejuizos-em-cidades-da-bahia.html >. Acessado em: 20 de jan. 2020. 
Identificação das regiões pluviométricas homogêneas e início e fim da estação chuvosa na Bacia do Médio São Francisco (Brasil)
Laurizio Emanuel Ribeiro Alves Heliofábio Barros Gomes

Washington Luiz Félix Correia Filho José Francisco de Oliveira Júnior Luis Gustavo Gonçalves de Gonçalves Dirceu Luis Herdies

Fabrício Daniel dos Santos Silva

GUEDES, R.V.S.; MACEDO, M.J.H.; SOUSA, F.A.S.DE. Análise espacial de eventos de secas com base no índice padronizado de precipitação e análise de agrupamento. Revista Brasileira de Ciências Ambientais, Rio de Janeiro - RJ, v.1, n. 23, p. 55-65, 2012.

INMET - Instituto Nacional de Meteorologia. Seção de Armazenamento de Dados Meteorológicos (SADMET). Estação Meteorológica de Observação de Superfície Automática. Disponível em: $<$ http://www.inmet.gov.br/projetos/rede/pesquisa/inicio.php>. Brasília: Acessado em 30 out. 2019.

KAYANO, M. T.; ANDREOLI, R. V. Clima da Região Nordeste do Brasil. In: Cavalcante, I. F. A. et al. (Org.) Tempo e Clima no Brasil. São Paulo: Oficina de Textos, 2009, p. 213-233.

KOUSKY, V. E. Pentad outgoing longwave radiation climatology for the South American sector. Revista Brasileira de Meteorologia, v. 3, n. 1, p. 217-231, 1988.ISSN 1982-4351.

LIEBMANN, B.; CAMARGO, S. J.; SETH, A.; MARENGO, J.A.; CARVALHO, L. M.; ALLURED, D.; FU, R.; VERA, C. S. Onset and End of the Rainy Season in South America in Observations and the ECHAM 4.5 Atmospheric General Circulation Model. Journal of Climate, v. 20, n. 10, p. 2037-2050, 2007.ISSN 1520-0442. https://doi.org/10.1175/JCLI4122.1

LIEBMANN; B.; MARENGO, J. A. Interannual Variability of the Rainy Season and Rainfall in the Brazilian Amazon Basin. Journal of Climate, v. 14, n.22, p.4308-4318, 2001.ISSN 1520-0442. https://doi.org/10.1175/1520-0442(2001)014<4308:IVOTRS>2.0.CO;2

LYRA, G. B.; OLIVEIRA-JÚNIOR, J. F.; GOIS, G.; CUNHA-ZERI, G.; ZERI, M. Rainfall variability over Alagoas undertheinfluencesof SST anomalies. Meteorology and Atmospheric Physics (Print), v. 129, p. 157-171, 2017. https://doi.org/10.1007/s00703-016-0461-1

LYRA, G. B.; OLIVEIRA-JÚNIOR, J. F.; ZERI, M. Cluster analysis applied to the spatial and temporal variability of monthly rainfall in Alagoas state, Northeast of Brazil. International Journal of Climatology, v. 34, p. 3546-3558, 2014. https://doi.org/10.1002/joc.3926

LYRA, M. J. A. Influência dos Processos Físicos e Sinóticos nas Trajetórias dos Complexos Convectivos de Mesoescala no Nordeste Brasileiro entre 2008 e 2015. 2018. 74 f. Dissertação (Mestrado em Meteorologia) - Universidade Federal de Alagoas, Maceió, 2018.

MAIA, R.; VIVAS, E.; SERRALHEIRO, R.; DE CARVALHO, M. Socioeconomic Evaluation of Drought Effects. Main Principlesand Application to Guadiana and Algarve Case Studies. Water Resources Management. v. 29, n. 2, p. 575-588, 2015. ISSN 0920-4741. https://doi.org/10.1007/s11269-014$\underline{0883-9}$

MARENGO, J. A.; ALVES, L. M.; ALVALA, R. C. S.; CUNHA, A. P.; BRITO, S.; MORAES, O. L.L. Climatic characteristics of the 2010-2016 drought in the semiarid Northeas tBrazil region. Anais da Academia Brasileira de Ciências, v. 90, n.2, p. 1973-1985, 2018

MARENGO, J. A.; CUNHA, A. P.; ALVES, L. M. A seca de 2012-15 no semiárido do Nordeste do Brasil no contexto histórico. Climanálise (Online), v. 3, p. 49-54, 2016.ISSN 0103-0019. https://doi.org/10.1590/0001-3765201720170206

MARENGO, J. A.; TORRES, R. R.; ALVES, L. M. Drought in Northeast Brazil-past, present, and future. Theoretical Applied and Climatology, 129: 1189-1200, $2017 \mathrm{https} / / / \mathrm{doi} .0 \mathrm{rg} / 10.1007 / \mathrm{s} 00704-$ 016-1840-8. https://doi.org/10.1007/s00704-016-1840-8

MARENGO, J. LIEBMANN, B.; KOUSKY, V.E.; FILIZOLA, N.P.; WAINER, I.C. Onset and End of the Rainy Season in the Brazilian Amazo nBasin. Journal of Climate, v. 14, n. 5, p. 833-852, 2001. ISSN 1520-0442.

MELO JÚNIOR, J.C.F. DE; SEDIYAMA, G.C.; FERREIRA, P.A.; LEAL, B.G. Determinação de regiões homogêneas quanto à distribuição de frequência de chuvas no leste do Estado de Minas Gerais. Revista Brasileira de Engenharia Agrícola e Ambiental, Campina Grande - PB, v. 10, n. 2, p. 4084016, 2006. https://doi.org/10.1590/S1415-43662006000200023 
Identificação das regiões pluviométricas homogêneas e início e fim da estação chuvosa na Bacia do Médio São Francisco (Brasil)
Laurizio Emanuel Ribeiro Alves Heliofábio Barros Gomes

Washington Luiz Félix Correia Filho

José Francisco de Oliveira Júnior Luis Gustavo Gonçalves de Gonçalves Dirceu Luis Herdies

Fabrício Daniel dos Santos Silva

MINGOTI, S. A. Análise de dados através de métodos de estatística multivariada: uma abordagem aplicada. Editora UFMG, 2007.

MMA - Ministério do Meio Ambiente. Secretaria dos Recurso Hídricos. Caderno da Região Hidrográfica do São Francisco- Ministério do Meio Ambiente, Secretária de Recursos Hídricos. Brasília: MMA, 2006. 148 p.

MOLION, L. C. B.; BERNARDO, S. O. Uma Revisão da Dinâmica das Chuvas no Nordeste Brasileiro. Revista Brasileira de Meteorologia, v. 17, n. 1, p. 1-10, 2002. ISSN 1982-4351.

MURAKAMI, T.; NAKAZAWA, T. Transpiration from the southern to northern hemisphere summer monsoon. Monthly Weather Review, v. 113, n. 9, p. 1470-1486, 1985. ISSN 0027-0644. https://doi.org/10.1175/1520-0493(1985)113<1470:TFTSTN>2.0.CO;2

OLIVEIRA-JÚNIOR, J. F.; XAVIER, F. M. G.; TEODORO, P. E.; GOIS, G.; DELGADO, R. C. Cluster Analysis Identified Rainfall Homogeneous Regions In Tocantins State, Brazil. Bioscience Journal (Online), v. 33, p. 333-340, 2017. https://doi.org/10.14393/BJ-v33n2-32739

PNUD - Programa das Nações Unidas para o Desenvolvimento. IPEA - Instituto de Pesquisas Aplicadas. FJP - Fundação João Pinheiro. CHEDIEK, J.; PERAL, A.; NERI, M. C.; CHAVES, M. (Org.). O Índice de Desenvolvimento Humano Municipal Brasileiro - Brasília: PNUD, IPEA, FJP, 2013. 96 p. (Atlas do Desenvolvimento Humano no Brasil 2013). Disponível em: < http://ipea.gov.br/portal/images/stories/PDFs/130729_AtlasPNUD_2013.pdf>. Acessado em $01 \mathrm{fev}$. 2020.

R DEVELOPMENT CORE TEAM. R. A language and environment for statistical computing. Vienna, Austria: R Foundation for Statistical Computing. ISBN 3-900051-07-0, 2017. Disponível em: $<\mathrm{http}: / / w w w . R-$ project.org/.>

RAO, V. B.; LIMA, M. C.; FRANCHITO, S. H. Seasonal and Interannual Variations of Rainfall over Eastern Northeast Brazil. Journal of Climate, v. 6, n. 9, p. 1754-1763, 1993.ISSN 1520-0442. https://doi.org/10.1175/1520-0442(1993)006<1754:SAIVOR>2.0.CO;2

REBOITA, M. S.; ASSIREU, A.T.; SILVA, L. C. DA; GAMARRA, N.L.R. Evidências de Circulação de Brisa Vale-Montanha na Serra da Mantiqueira: Cidade de Itajubá - MG. Ciência e Natura, v. 36, n.1, p. 61-71, 2014. ISSN 0100-8307. https://doi.org/10.5902/2179460X12876

REBOITA, M. S.; GAN, M. A.; ROCHA, R. P. D.; AMBRIZZI, T. Regimes de Precipitação na América do Sul: Uma revisão Bibliográfica. Revista Brasileira De Meteorologia. v. 25, n.1, p. 185-204, 2010. ISSN 1982-4351. https://doi.org/10.1590/S0102-77862010000200004

RIBEIRO FILHO, N. P. Melhorando o desempenho da técnica de clusterização hierárquica Single Linkage utilizando Metaheurística GRASP. 2016. 59 f. Dissertação (Mestrado em Modelagem Computacional de Sistemas) - Universidade Federal de Tocantins, 2016.

ROHLF, F. J. Adaptative hierarquical clustering schemes. Systematic Zoology, v.19, p.58-82, 1962. ISSN 1076-836X. https://doi.org/10.1093/sysbio/19.1.58

SANTOS, L. F.; GARCIA, S. R. Início e Fim da Estação Chuvosa no Estado de Minas Gerais: Comparação de Duas Metodologias Diferentes. Revista Brasileira de Meteorologia, v. 31, n.1, p. 92104, 2016. ISSN 1982-4351. https://doi.org/10.1590/0102-778620150017

SANTOS, W. M. DOS; SOUZA, R. M. S.; SOUZA, E. S. DE; ALMEIDA, A. Q. DE; ANTONINO, A. C. D. Variabilidade espacial da sazonalidade da chuva no semiárido brasileiro. Journal of Evironmental Analysis and Progress, v. 2, n. 4, p. 368-376. 2017. https://doi.org/10.24221/jeap.2.4.2017.1466.368$\underline{376}$

SILVA, D. F.; BRITO, J. I. B. Variabilidade do vento na bacia hidrográfica do rio São Francisco durante a ocorrência da ZCAS. Ambiência, Guarapuava, PR, v. 4, n. 2, p. 221-235, 2008. ISSN 2175-9405. 
Identificação das regiões pluviométricas homogêneas e início e fim da estação chuvosa na Bacia do Médio São Francisco (Brasil)
Laurizio Emanuel Ribeiro Alves Heliofábio Barros Gomes Washington Luiz Félix Correia Filho José Francisco de Oliveira Júnior Luis Gustavo Gonçalves de Gonçalves Dirceu Luis Herdies Fabrício Daniel dos Santos Silva

SILVA, F. C.; CRUZ, M. L. B. Análise da fisionomia da cobertura vegetal em ambientes semiáridos: o caso do município de Jaguaretama, estado do Ceará. Revista de Geociências do Nordeste. Caicó, v. 2, n. Especial, p. 801-809. 2017. ISSN 2447-3359.

SUGAHARA, S. Flutuações interanuais, sazonais e intrasazonais de precipitação no estado de São Paulo. 1991. 140 f. Tese (Doutorado em Meteorologia) -Universidade de São Paulo, São Paulo, 1991.

VILLARON, M. A.; FISCH, G. F. Caracterização do início do período chuvoso no Vale do Paraíba paulista. Ambiente \& Água - An Interdisciplinary Journal of Applied Science, v. 8, n. 1, p. 36-49, 2013.ISSN 1980-993X. https://doi.org/10.4136/ambi-agua.1254

WARD, J. H. Hierarchical grouping to optimize an objective function. Journal of the American Statistical Association, v. 58, n. 301, p. 236-244, 1963. ISSN 1537-274X. https://doi.org/10.1080/01621459.1963.10500845

WILHITE, D. A. Drought monitoring as a component of drought preparedness planning. In: IGLESIAS, A., GARROTE, L., CANCELLIERE, A., CUBILLO, F., WILHITE, D. (Orgs.). Coping with Drought Risk in Agriculture and Water Supply Systems. Springer Netherlands, 2009. cap. 1, p. 3-19. https://doi.org/10.1007/978-1-4020-9045-5 1

Recebido em: 24/05/2020

Aceito para publicação em: 23/09/2021 